\title{
UTILIZACION DEL NITROGENO EN DIFERENTES MANEJOS DE FERTILIZACIÓN EN LECHUGA (Lactuca sativa L.) Y SU EFECTO SOBRE ALGUNAS VARIABLES BIOLÓGICAS DEL SUELO, NEUQUEN - ARGENTINA.
}

\author{
María Cristina Aruani ${ }^{1}$, Perla Gilii ${ }^{1}$, Lidia Fernández ${ }^{2}$, Ricardo González Junyent ${ }^{1}$, Pablo \\ Reeb $^{1}$, Enrique Sánchez ${ }^{3}$ \\ ${ }^{1}$ Universidad Nacional del Comahue (UNCo), Facultad de Ciencias Agrarias, Ruta 151, km 12,5, \\ Cinco Saltos, (C.P.8303) Río Negro, Argentina (mcaruani@jetband.com.ar) \\ ${ }^{2}$ Universidad Nacional del Comahue (UNCo), Escuela Superior de la Salud y el Ambiente, Buenos \\ Aires 1400 (C.P. 8300) Neuquén, Argentina. \\ ${ }^{3}$ Estación Experimental Agropecuaria Alto Valle de Río Negro, General Roca, (C.P. 8332) Río \\ Negro, Argentina.
}

\section{ABSTRACT \\ Nitrogen utilization of different fertilization regimes in lettuce (Lactuca sativa $\mathrm{L}$.) and its efect on biological properties of the soil on Neuquén, Argentina.}

Key words: organic amendment, nitrogen fertilization, nitrogen use efficiency, biological indexes.

The goal of this work was to determine the efficiency of nitrogen use (EUN) in a lettuce crop fertilized with organic and inorganic $\mathrm{N}$ sources and to evaluate its effect on some biological properties of the soil.

Treatments were: 1) Control (no N; No), inorganic $\mathrm{N}$ (100 kg ha-1 $\mathrm{N}$ applied as ammonium nitrate; Fi) and organic $\mathrm{N}$ (chicken manure equivalent to a rate of $100 \mathrm{~kg} \mathrm{~N} \mathrm{ha}^{-1}$; E) in a complete randomized block design with five replications. In the soil nitrate levels at the $0-30$; 30-60 and 60-90 cm depths, the indices of carbon mineralization, microbial carbon biomass (C-BM) and the soil respiration coefficient (q $\mathrm{CO} 2$ ) were determined with each treatment. Total yield was 49.1; 34.6 and $24.7 \mathrm{t} /$ ha for treatments E, Fi and No, respectively. The EUN was 25 and $16 \%$ for the $\mathrm{E}$ and Fi treatments, respectively. The C-BM and RE were higher in the fertilized treatments.

\section{RESUMEN}

Palabras claves: enmienda orgánica, fertilización nitrogenada, uso eficiente del nitrógeno, indicadores biológicos.

El objetivo del ensayo fue determinar la eficiencia de utilización del nitrógeno en un cultivo de lechuga con aporte de nitrógeno en forma orgánica y mineral y evaluar su efecto en el suelo a través de variables químicas, biológicas y enzimáticas.

Los tratamientos fueron: testigo $\left(\mathrm{N}_{0}\right) \sin$ fertilizar, fertilización química $100 \mathrm{~kg} \mathrm{hg} \mathrm{ha-1}$ de $\mathrm{N}$ aplicado como nitrato de amonio (Fi) y fertilización con enmienda orgánica, estiércol de pollo (E), donde se agregó una cantidad equivalente a $100 \mathrm{~kg} \mathrm{ha}^{-1}$ de $\mathrm{N}$. El diseño experimental fue en bloques completamente aleatorizados con tres tratamientos y tres repeticiones. Se determinó la concentración de nitratos a 0-30, 30-60 y 30-60 cm de profundidad del suelo y se calcularon los índices de mineralización del carbono, proporción de $\mathrm{C}$ de la biomasa microbiana $(\mathrm{C}-\mathrm{BM})$ en la $\mathrm{MO}$, respiración edáfica $(\mathrm{RE}) \mathrm{y}$ coeficiente respiratorio del suelo $\left(\mathrm{qCO}_{2}\right)$. Los rendimientos en $\mathrm{t} \mathrm{ha}^{-1}$ en los tratamientos $\mathrm{E}$, Fi y $\mathrm{N}_{0}$ fueron de 49,1, 34,6 y 24,7 respectivamente. La eficiencia de utilización de nitrógeno fue del $25 \%$ en $\mathrm{E}$ y $16 \%$ en Fi. Al final del cultivo se obtuvo diferencias entre C-BM y los tratamientos, siendo mayores en $\mathrm{E}$ y Fi con respecto a $\mathrm{N}_{0}$. La respiración siguió el mismo comporta-miento que el C-BM.

Recepción de originales: 7 de abril de 2008. 


\section{INTRODUCCIÓN}

En los valles de la Patagonia Norte (Río Negro y Neuquén), la horticultura ha registrado una constante evolución y en la actualidad se estima que supera las 8.000 ha, ello representa un incremento de aproximadamente el $65 \%$ en los últimos diez años (Iglesias et al., 2002).

El nitrógeno $(\mathrm{N})$ es el nutriente con mayor impacto sobre el rendimiento y la calidad de los cultivos hortícolas en general. Es extremadamente dinámico en el suelo y sufre cambios que incluyen procesos de pérdidas, ganancias y transformaciones.

El exceso de $\mathrm{N}$ en la fase de crecimiento de la lechuga origina un crecimiento desordenado, con hojas excesivamente grandes y frágiles que dificulta el manejo (Maroto, 2002). Se recomienda fertilizar el cultivo con 100 y $200 \mathrm{~kg}$ $\mathrm{ha}^{-1}$ de $\mathrm{N}$ disponible en la zona radical (Sorensen et al., 1994) y la cantidad de $\mathrm{N}$ disponible para la planta depende directamente del manejo del agua en distintos sistemas de riego (Cantliffe et al., 1998). Cuando ésta es insuficiente la absorción del nitrógeno y los rendimientos disminuyen marcadamente y si es excesiva provoca lavado y pérdida de nitrógeno (Karam et al., 2002). En la zona norpatagónica no existen limitaciones para la disponibilidad de agua y el manejo del riego es muy deficitario (Draghi, 2005; Aruani et al., 2007). La modalidad de riego de los productores se ajusta a los turnos de riego, sin tener en cuenta las necesidades de agua del cultivo, por ello, generalmente es excesiva.

En el país es ampliamente estudiada la respuesta de nitrógeno en cereales (Echeverría y Videla, 1998, Echeverría y Sainz Rozas, 2001) pero el conocimiento entre el régimen de agua sobre el rendimiento y la absorción de nitrógeno es escaso en horticultura y en mayor medida en la región Norpatagónica.

En este marco y con esta problemática es necesario cuantificar las eficiencias que describen la utilización del nitrógeno y la fisiológica. La eficiencia de utilización del nitrógeno aplicado (EUN), también llamado coeficiente de utilización del nitrógeno (Machet et al., 1987), representa la fracción de nitrógeno incorporado al suelo que es absorbido por el cultivo y depende de las propiedades del sistema radical, su distribución en el suelo, el área superficial, de la absorción por unidad de área y del manejo (Novoa y Loomis, 1981). El índice EUN puede determinarse por el método de la diferencia, que es uno de los más utilizados. La eficiencia fisiológica (EF), en tanto, representa la cantidad de materia seca producida por unidad de nitrógeno absorbido (kg de materia seca por kg de nitrógeno absorbido). Con esta información y el conocimiento del nitrógeno remanente en el suelo después de la cosecha del cultivo, es factible la confección del balance del nitrógeno (Hofman y van Cleemput, 2004). Estos estudios conducen a mejorar la eficiencia de uso del nitrógeno aportado por fertilización.

La dinámica en la disponibilidad de nutrientes es diferente al incorporar compuestos orgánicos en el manejo de los suelos hortícolas, respecto a los sistemas que reciben una fuente mineral. La calidad de la materia orgánica aportada depende de los materiales de origen y del grado de estabilización; materiales con alto contenido de materia orgánica lábil inducen mayor actividad biológica y liberación de nutrientes en el suelo, mientras que la materia orgánica de lenta descomposición con alta relación $\mathrm{C} / \mathrm{N}$ contribuye a mejorar la capacidad de almacenamiento de agua y nutrientes y a controlar la erosión (Cooperband, 2000).

Los procesos de descomposición son llevados a cabo por los microorganismos edáficos, por ello, la actividad biológica del suelo resulta de importancia fundamental para entender las modificaciones que se producen al agregar residuos orgánicos (Lupwayi et al., 1998: Abril, 2003), ó fertilizantes químicos. Estudios de Lal (1998) y Arshad y Martín (2002) indicaron que los parámetros biológicos, en periodos cortos de tiempo, son más sensibles que el contenido de materia orgánica (MO) de un suelo dado, ya que la MO varía muy lentamente y sólo es posible detectar los cambios a largo plazo. Algunos autores sugieren que el cálculo de índices relacionando diferentes variables (biomasa/ respiración, biomasa/materia orgánica) permiten determinar la calidad del suelo (Filip, 2002).

La calidad del suelo es un criterio clave para evaluar prácticas agronómicas adecuadas, que posibilitan mantener la sostenibilidad del recurso. Sin embargo, en el Alto Valle de Río Negro 
y Neuquén no se cuenta con información que permita establecer el balance de nitrógeno y los efectos de las enmiendas orgánicas y prácticas asociadas a la fertilización química, sobre la sostenibilidad de los ecosistemas hortícolas.

El objetivo del ensayo fue determinar la eficiencia de utilización del nitrógeno en un cultivo de lechuga con aporte de nitrógeno en forma orgánica y mineral y evaluar su efecto en el suelo a través de variables químicas, biológicas y enzimáticas

\section{MATERIALES Y MÉTODOS}

El ensayo se realizó en condiciones de campo en una chacra comercial de varios años de trayectoria hortícola, ubicada en la localidad de Centenario, provincia del Neuquén (LS $38^{\circ} 52^{\circ}$ 33,1” y LO 68 05‘ 8,4”). El cultivo utilizado fue lechuga (Lactuca sativa L.) var Maravilla Cuatro Estaciones.

El suelo en estudio corresponde al orden Aridisol clasificado como Haplocambid típico (Soil Survey Staff, 1998), de textura franca limoso. A los $30 \mathrm{~cm}$ de profundidad el $\mathrm{pH}$ fue de 7,6, el contenido de materia orgánica (MO) (Walkey y Black, 1934) de $30 \mathrm{~g} \mathrm{~kg}^{-1}$, nitrógeno total (Nt) de $1,1 \mathrm{~g} \mathrm{~kg}^{-1}$, fósforo disponible (Olsen, 1934) de $14,7 \mathrm{mg} \mathrm{kg}^{-1}$ y potasio intercambiable $0,9 \mathrm{cmol}_{\mathrm{c}} \mathrm{kg}^{-1}$.

En la primavera del 2006, se utilizaron 9 parcelas de $4 m^{2}(2 m \times 2 m)$ como unidades experimentales y se instalaron los siguientes tratamientos en un diseño en bloques completamente aleatorizado: testigo sin fertilizar $\left(\mathrm{N}_{0}\right)$; fertilización química, $100 \mathrm{~kg} \mathrm{ha}^{-1}$ de nitrógeno aplicado como nitrato de amonio (Fi) y fertilización con enmienda orgánica, estiércol de pollo (E), donde se agregó una cantidad equivalente a $100 \mathrm{~kg} \mathrm{ha}^{-1}$ de nitrógeno $(\mathrm{N})$.

La aplicación del estiércol se realizó el 8 de septiembre.

En el Cuadro 1 se detalla el análisis químico del estiércol empleado, el valor de fósforo es el disponible determinado por el método Olsen, y el $\mathrm{K}$ es el intercambiable extraído con acetato de amonio

La fertilización química fue aplicada en dos dosis iguales, a los 7 y 19 días posteriores al transplante (23 de octubre y 4 de noviembre respectivamente).

Los plantines de lechuga se obtuvieron en semillero y se transplantaron el 16 de octubre a una distancia de $0,40 \mathrm{~m}$ colocados a doble fila por platabanda, esto representa un total de 36 plantas por parcela (75000 pl ha-1). Se utilizó como unidad de observación las 10 plantas centrales, para evitar el efecto de borde y la cosecha se realizó cuando el cultivo alcanzó el desarrollo comercial (5 de diciembre).

Se tomaron muestras de suelo en cada tratamiento; cada una de ellas compuesta de 6 submuestras a diferentes profundidades (0-30, $30-60,60-90 \mathrm{~cm}$ ) y tiempo, antes del agregado del estiércol (datos considerados iniciales), después de cada fertilización inorgánica (4 y 18 de noviembre), y antes de cosecha (3 de

Cuadro 1. Propiedades del estiércol de pollo utilizado en el ensayo, (promedio de 5 repeticiones).

Los resultados están expresados en base de materia seca.

Table 1. Properties of the chicken manure used on the experiment (mean of 5 replications, dry matter basis).

\begin{tabular}{|c|c|c|c|c|c|c|c|}
\hline & \multirow{3}{*}{$\mathrm{pH}$} & \multirow{3}{*}{$\begin{array}{c}\mathrm{CE} \\
(\mathrm{dS} / \mathrm{m})\end{array}$} & CO & N & \multirow{3}{*}{$\mathrm{C} / \mathrm{N}$} & \multirow{3}{*}{$\begin{array}{c}\mathrm{P} \\
\text { disponible } \\
(\%)\end{array}$} & \multirow{3}{*}{$\begin{array}{c}\mathrm{Ki} \\
\left(\mathrm{cmol}_{\mathrm{c}} \mathrm{kg}^{-1}\right)\end{array}$} \\
\hline & & & 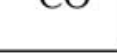 & Not & & & \\
\hline & & & \multicolumn{2}{|c|}{$\mathrm{g} \mathrm{kg}^{-1}$} & & & \\
\hline Promedio & 6,8 & 17 & 458 & 28 & 16,3 & 0,3 & 45 \\
\hline $\begin{array}{c}\text { Desviación } \\
\text { estándar }\end{array}$ & $\pm 0,1$ & $\pm 0,02$ & $\pm 12,6$ & $\pm 1,2$ & & $\pm 0,1$ & $\pm 7,1$ \\
\hline
\end{tabular}

CE: conductividad eléctrica; $\mathrm{CO}$ : carbono orgánico; Nt: nitrógeno total; P: fósforo; Ki: potasio intercambiable y \pm : desvío estándar 
diciembre). Debe tenerse en cuenta que el 4 de noviembre se extrae la muestra y se aplica la segunda dosis de fertilizantes. Se determinó la concentración de nitratos $\left(\mathrm{NO}_{3}^{-}\right)$por un equipo de medición rápida (Merck reflectoquant), que expresa el resultado directamente en $\mathrm{mg} \mathrm{kg}^{-1}$ de $\mathrm{NO}_{3}^{-1}$. Este valor se corrigió por la humedad del suelo al momento de la extracción.

Se evaluó materia fresca foliar (MFf), luego las hojas fueron secadas a $75{ }^{\circ} \mathrm{C}$ hasta peso constante, pesadas y molidas para determinar la materia seca foliar (MSf). En esta muestra se determinó $\mathrm{N}$ total $(\mathrm{N})$, fósforo $(\mathrm{P})$, potasio $(\mathrm{K})$ y calcio (Ca).

Con la información obtenida se determinó el rendimiento: $k g$ MFf ha- ${ }^{-1}$ y $\mathrm{kg} \mathrm{MSf} \mathrm{ha}^{-1}$, nitrógeno foliar absorbido $\mathrm{kg} \mathrm{ha}^{-1} \mathrm{y}$ eficiencia fisiológica (EF), definida como la relación entre el rendimiento MSf y el nitrógeno absorbido (Hofman y van Cleemput, 2004) y la eficiencia de utilización del nitrógeno (EUN) definida como la fracción de nitrógeno aplicado que es absorbido por el cultivo, determinado por el método indirecto EUN $=(\mathrm{NPF}-\mathrm{NPT}) \times 100$ / DN. Donde NPF= nitrógeno acumulado en la biomasa foliar del tratamiento fertilizado, NPT= nitrógeno acumulado en la biomasa foliar de las plantas testigo y $\mathrm{DN}=$ dosis de nitrógeno agregada (Machet et al., 1987).

El riego fue gravitacional, modalidad utilizada por los productores de la zona y se midió la lámina de agua aplicada en el momento del transplante, en noviembre y cerca de cosecha utilizando un aforador de régimen crítico trapezoidal para surco, localizado en la acequia de riego de las parcelas.

El 8 de setiembre, antes de agregar estiércol y a la cosecha se extrajeron muestras de suelo de $0-15 \mathrm{~cm}$, con el propósito de medir las siguientes variables químicas y biológicas Determinaciones químicas: nitrógeno total $(\mathrm{Nt})$, (Bremmer y Mulvaney, 1982); carbono orgánico total (COT), mediante el método de (Walkley y Black). Los resultados se expresaron en $\mathrm{mg}$ $\mathrm{C} \mathrm{kg}^{-1}$ de suelo (Nelson y Sommers, 1982). Determinaciones biológicas: respiración edáfica (RE), por incubación controlada durante 10 días determinando el $\mathrm{CO}_{2}$ capturado en $\mathrm{NaOH}$ 0,1 M, titulando con $\mathrm{HCl}$ 0,1 M (Weaver et al.,
1994), los resultados se expresaron en mg $C 100$ $\mathrm{g}^{-1}$ suelo seco de suelo; carbono de la biomasa microbiana (C-BM), utilizando el método de la respiración inducida por sustrato (SIR) (Anderson y Domsch, 1978; Öhlinger, 1996), los resultados se expresaron en mg C $100 \mathrm{~g}^{-1}$ suelo seco; coeficiente respiratorio $\left(\mathrm{qCO}_{2}\right)(\mathrm{Paul}$ y Clark, 1996; Frioni, 1999) (cociente entre RE y C-BM); índice de mineralización del carbono (IM) (cociente entre RE y materia orgánica (MO) (Abril y Bucher, 2001); proporción de C de la biomasa microbiana en la MO del suelo (cociente entre C-BM y COT) (Carter, 1991); actividad deshidrogenasa (Dh-asa) por reducción de 2,3,5 cloruro de tetrazolio a trifenil formazan (TPF) y detectada por espectrofotometría (Weaver et al., 1994), los resultados se expresaron en $\mu \mathrm{g}$ 2,3,5 TPF g ${ }^{-1}$ suelo $\mathrm{h}^{-1}$; actividad catalasa (C) (Jonson y Temple, 1964), se determinó mediante la cantidad de $\mathrm{H}_{2} \mathrm{O}_{2}$ que fue transformada después de ser agregada al suelo, los resultados se expresaron en $\mu \mathrm{mol} \mathrm{H}_{2} \mathrm{O}_{2}$ transformada $\mu \mathrm{g}^{-1}$ suelo $\mathrm{min}^{-1}$. Todos los estudios químicos, biológicos y enzimáticos se realizaron por triplicado.

\section{Análisis de la información}

Para estudiar la evolución de los nitratos se consideraron modelos con distintas estructuras de covarianzas a fin de ajustar la dependencia espacio-temporal (profundidad-fecha de muestreos) de las unidades experimentales (Littel et al. 2006). También se consideró la inclusión de la covariable concentración de nitrato inicial para explicar la situación de partida de cada parcela.

Los rendimientos y los contenidos de N, P, Ca y $\mathrm{K}$ foliar fueron evaluados a través de un análisis de varianza (ANDEVA).

Las variables biológicas se analizaron por el método de varianza (ANDEVA), unifactorial por fecha y se realizó una correlación simple entre dichas variables (C-BM, C y Dh-asa) y el contenido nitratos en el suelo.

Para la comparación de medias se utilizó el test de mínima diferencia significativa (LSD) con un nivel de significancia de 0,05 . 
Cuadro 2. Concentración media de nitratos en suelo para los diferentes tratamientos y profundidad Table 2. Mean soil nitrate concentration for the different treatments and soil depths

\begin{tabular}{|c|c|c|c|}
\hline \multirow{4}{*}{ Fechas } & \multicolumn{3}{|c|}{ Tratamientos } \\
\hline & No & $\mathrm{Fi}$ & E \\
\hline & \multicolumn{3}{|c|}{$\mathrm{mg} \mathrm{kg-1}$} \\
\hline & \multicolumn{3}{|c|}{$0-30(\mathrm{~cm})$} \\
\hline 08 -sept & $43,3(4,4)$ & $97,4(48,7)$ & $109,0(49,3)$ \\
\hline 04-nov & $35,7(8,4)$ & $148,7(71,9)$ & $191,3(62,4)$ \\
\hline 18-nov & $21,3(4,2)$ & $101,8(6,5)$ & $35,8(10,8)$ \\
\hline \multirow[t]{2}{*}{ 03-dic } & $20,6(2,3)$ & $30,7(10,0)$ & $26,9(8,5)$ \\
\hline & \multicolumn{3}{|c|}{$30-60(\mathrm{~cm})$} \\
\hline 08-sept & $38,9(10,7)$ & $83,2(44,0)$ & $126,7(46,5)$ \\
\hline 04-nov & $30,7(3,7)$ & $115,1(9,7)$ & $279,0(119,6)$ \\
\hline 18-nov & $11,3(3,8)$ & $46,4(13,2)$ & $19,4(9,4)$ \\
\hline \multirow[t]{2}{*}{ 03-dic } & $9,3(1,5)$ & $10,0(3,2)$ & $15,6(5,4)$ \\
\hline & \multicolumn{3}{|c|}{$60-90(\mathrm{~cm})$} \\
\hline 08-set & $19,5(4,3)$ & $86,1(54,1)$ & $98,0(34,5)$ \\
\hline 04-nov & $25,4(7,0)$ & $46,7(22,2)$ & $206,0(99,2)$ \\
\hline 18-nov & $9,1(1,2)$ & $30,1(5,4)$ & $8,3(2,5)$ \\
\hline 03-dic & $9,0(1,5)$ & $9,9(2,8)$ & $11,5(5,3)$ \\
\hline
\end{tabular}

El valor entre paréntesis indica el error estándar

\section{RESULTADOS}

Monitoreo del contenido de nitratos en el suelo

En el análisis de varianza de la concentración de nitratos, la interacción de tercer orden (tratamiento por profundidad por fecha) y la de segundo orden debida a profundidad por fecha no resultaron significativas, mientras que las correspondientes a tratamiento por fecha y tratamiento por profundidad resultaron significativas $(p=0,0063$ y $p=0,047$, respectivamente). En el Cuadro 2 se observa la distribución de los nitratos en los diferentes tratamientos para cada fecha y profundidad. La variabilidad observada al comienzo del ensayo (8 de septiembre) es consecuencia de la historia hortícola de la parcela y al modo de fertilización orgánica que realiza el productor en la franja de plantación. Si bien esta variabilidad fue importante no fue estadísticamente significativa cuando se incluyó como covariable. En la segunda fecha de muestreo, se determinó que la concentración fue significativamente mayor en el tratamiento E. Estos valores pueden explicarse dado que el estiércol fue aplicado en el momento de preparación del terreno, contando con un tiempo suficiente para su descomposición. A partir de la tercera fecha de muestreo, la concentración de nitratos disminuye notablemente en la parcela $\mathrm{E}$ o eliminar con valores semejantes a $\mathrm{N}_{0}$ (menores a $50 \mathrm{mg}$ $\mathrm{kg}^{-1}$ ), mientras que en las parcelas Fi duplican dicho valor. Al finalizar el ciclo de cultivo, no se observa diferencia entre los tratamientos, los valores de nitratos son semejantes en todas las parcelas.

Al realizar el análisis en función de la profundidad, se observa un incremento significativo en la concentración de nitratos a $30 \mathrm{~cm}$ en $\mathrm{Fi}$, mientras que en $\mathrm{E}$ se distribuyen hasta los $60 \mathrm{~cm}$.

\section{Producción del cultivo}

En el Cuadro 3 se informan los rendimientos de materia fresca obtenidos en los diferentes tratamientos y concentraciones de macronutrientes en la biomasa foliar. Se obtuvo diferencia en los rendimientos de MFf por ha en el

Cuadro 3. Rendimiento en fresco del cultivo de lechuga y concentración de macronutrientes en la biomasa foliar seca en los diferentes tratamientos

Table 3. Fresh yield of lettuce crop and macronutrient concentrations of the dry foliar biomass for the different treatments.

\begin{tabular}{c|c|c|c|c|c}
\hline \multirow{2}{*}{ Tratamientos } & Rendimiento & Nitrógeno & Fósforo & Potasio & Calcio \\
\cline { 2 - 6 } & $\left(\mathrm{t} \mathrm{ha}^{-1}\right)$ & \multicolumn{4}{|c}{$(\%)$} \\
\hline $\mathrm{N}_{0}$ & $24,7 \mathrm{~b}$ & $2,3 \mathrm{~b}$ & 0,47 & 7,14 & 0,84 \\
$\mathrm{Fi}$ & $34,6 \mathrm{~b}$ & $2,8 \mathrm{a}$ & 0,42 & 7,36 & 1,02 \\
$\mathrm{E}$ & $49,1 \mathrm{a}$ & $2,7 \mathrm{a}$ & 0.44 & 7,60 & 1,01 \\
\hline
\end{tabular}

(Letras diferentes en cada columna significa diferencia significativa en cada tratamiento) 
tratamiento con estiércol $(\mathrm{E})$ respecto al testigo $\left(\mathrm{N}_{0}\right)(\mathrm{p}=0,0034)$ y al fertilizante químico $(\mathrm{Fi})$ $(p=0,02)$, esto corresponde en $t h^{-1}$ en los tratamientos fertilizados de 49,1 y 34,6 en E y Fi respectivamente y 24,7 en el testigo. De acuerdo al análisis de varianza de los contenidos foliares de los macronutrientes se obtuvo solamente diferencia significativa en nitrógeno.

\section{Eficiencia de utilización del nitrógeno}

Los datos del Cuadro 4 muestran la eficiencia de utilización del nitrógeno (EUN) de la biomasa aérea y parámetros relacionados. Al momento de la cosecha (49 días posteriores al transplante) el nitrógeno promedio total absorbido por la

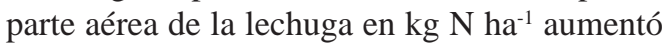
con el aporte de $\mathrm{N}$ orgánico. La eficiencia de utilización del nitrógeno aplicado (EUN) (Machet et al., 1987) cambió significativamente en los tratamientos fertilizados, en el tratamiento con estiércol se utilizó el 25\% y mientras que con nitrógeno mineral fue del $16 \%$.

Al realizar el análisis de correlación entre rendimiento y la concentración de nitrato y el rendimiento y el nitrógeno foliar se obtuvo una relación positiva de $\mathrm{r}=0,86(\mathrm{p}=0,003)$ y $\mathrm{r}=0,75$ $(p=0,02)$ respectivamente.

\section{Monitoreo de las variables biológicas}

Los valores promedio de la variable COT no mostraron diferencias entre los tratamientos al inicio y final del cultivo. Las magnitudes en ambas fechas, oscilaron entre 14 y $21 \mathrm{mg} \mathrm{C} \mathrm{kg}^{-1}$.
En el Cuadro 5 se observan los valores medios del C-BM, Dh-asa, C y respiración. Al final del cultivo se obtuvo diferencias entre' $\mathrm{C}$-BM $y$ los tratamientos $(p=0,0002)$. Se observa un incremento positivo en las parcelas fertilizadas, el mejor comportamiento se muestra en las parcelas tratadas con estiércol (E). Estas diferencias fueron mayores respecto a las testigos $\left(\mathrm{N}_{0}\right)(\mathrm{p}=0,002)$ y a las fertilizadas con nitrógeno inorgánico (Fi) $(\mathrm{p}=0,015)$ y Fi respecto a $\mathrm{N}_{0}(\mathrm{p}=0,04)$. La respiración sigue el mismo comportamiento que el C-BM. El C-BM entre las fechas analizadas no presentó diferencias significativas debido al tiempo transcurrido (85 días). En un trabajo realizado con manzano se observó que el efecto de la fertilización nitrogenada sobre la actividad microbiana se produce aproximadamente entre los 20-25 días de aplicado el fertilizante (Gili, et al. 2007).

La medición de la actividad de la Dh-asa, que constituye una medida de la actividad promedio de la población de microorganismos viables, no evidenció diferencias significativas al final del cultivo. Similar comportamiento se observó en la catalasa.

En el Cuadro 6 se muestran los resultados de las combinaciones de las variables químicas y biológicas (índices). Los resultados del cociente metabólico $\left(\mathrm{qCO}_{2}\right)$ se mantienen prácticamente constantes en todos los tratamientos y épocas de muestreo, los valores promedios fueron 0,05. La relación C-BM/COT, índice que indica la

Cuadro 4. Eficiencia de utilización del nitrógeno y sus parámetros asociados.

Table 4. Efficiency of nitrogen use and associated parameters.

\begin{tabular}{c|c|c|c|c|c}
\hline Tratamientos & $\begin{array}{c}\text { N agregado } \\
\left(\mathrm{kg} \mathrm{ha}^{-1}\right)\end{array}$ & $\begin{array}{c}\text { MSf } \\
\left(\mathrm{kg} \mathrm{ha}^{-1}\right)\end{array}$ & $\begin{array}{c}\mathrm{N} \\
\text { absorbido } \\
\left(\mathrm{kg} \mathrm{ha}^{-1}\right)\end{array}$ & EF (1) & EUN (\%) (2) \\
\hline $\mathrm{N}_{0}$ & 0 & 1442 & $33,1 \mathrm{~b}$ & 43,6 & \\
\hline $\mathrm{Fi}$ & 100 & 1754 & $49,6 \mathrm{~b}$ & 35,3 & 16 \\
\hline $\mathrm{E}$ & 100 & 2179 & $58,6 \mathrm{a}$ & 37,2 & 25 \\
\hline
\end{tabular}

(1) Eficiencia fisiológica, (MSf/N absorbido)

(2) Eficiencia de utilización del Nitrógeno, ( $\mathrm{N}$ absorbido del fertilizante $-\mathrm{N}$ absorbido testigo)/ $\mathrm{N}$ agregado) $* 100$

(letras diferentes en cada columna significa diferencia significativas en cada tratamiento) 
Cuadro 5. Valores medios de C-biomasa, respiración, actividad de la deshidrogenasa y catalasa en los diferentes tratamientos y fechas de muestreos.

Table 5. Mean values for C-biomass, respiration, deshidrogenase and catalase activities for each treatment and sampling date

\begin{tabular}{|c|c|c|c|c|c|c|c|c|}
\hline \multirow[t]{2}{*}{$\begin{array}{l}\text { Trata- } \\
\text { mientos }\end{array}$} & \multicolumn{2}{|c|}{ 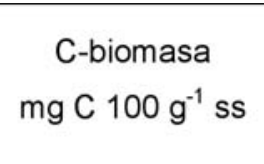 } & \multicolumn{2}{|c|}{$\begin{array}{c}\text { Respiración } \\
\mathrm{mg} \mathrm{C} 100 \mathrm{~g}^{-1} \mathrm{ss}\end{array}$} & \multicolumn{2}{|c|}{$\begin{array}{c}\text { Deshidrogenasa } \\
\mu 2,3,5 \mathrm{TPF}^{-1}\end{array}$} & \multicolumn{2}{|c|}{$\begin{array}{r}\text { Catalasa } \\
\mu \mathrm{mol} \mathrm{H}_{2} \mathrm{O}_{2} \\
\text { ansformada g }{ }^{-1}\end{array}$} \\
\hline & Inicial $^{\star}$ & final & inicial & final & inicial & final & inicial & final \\
\hline $\mathrm{N}_{0}$ & 100,6 & $96,6 c^{\star}$ & 6,1 & $4,7 \mathrm{c}$ & 0,43 & 1,46 & 19,2 & 23,3 \\
\hline $\mathrm{Fi}$ & 128,9 & $112,4 b$ & 8,1 & $5,4 b$ & 0,78 & 1,26 & 18,8 & 24,5 \\
\hline E & 107,4 & 133,6 a & 6,9 & $6,5 a$ & 0,74 & 1,72 & 20,5 & 25,7 \\
\hline
\end{tabular}

(* Letras diferentes significan deferencias significativas en cada tratamiento)

* Inicial: 8 de setiembre; Final: 3 de diciembre

eficiencia metabólica de la biota, varió entre 5 y 7 . Aunque, no se encontraron diferencias significativas en las fechas de muestreos, se observa un incremento de esta relación hacia el final del cultivo en el tratamiento E.

El índice de mineralización (IM) fue alto al inicio del cultivo, con valores superiores a 2. Al finalizar el ciclo, los valores de este índice descendieron por debajo de 2 en los tratamientos $\mathrm{N}_{0}$ y Fi, sin llegar a valores cercanos a 1, valor que indica un balance equilibrado entre mineralización y humificación del carbono. En el tratamiento E, el índice se desplazó hacia valores superiores a 2.

\section{DISCUSION}

\section{Concentración de nitratos en el suelo}

Diversos autores han reflejado aumentos de la concentración de nitratos en la solución del suelo durante las primeras etapas vegetativa de la lechuga cuando no se producen pérdidas altas por lixiviación y las cantidades de $\mathrm{N}$ consumidas son mínimas (Jackson et al., 1993; Mc Pharlin et al., 1995). Los resultados de la presente experiencia son coincidentes con lo expuesto anteriormente, atribuyendo el aumento en la concentración de nitratos al exceso del $\mathrm{N}$ aportado en relación a lo consumido por el cultivo en la primera fase

Cuadro 6. Promedios del índice de mineralización (IM) y la relación CBM/COT en los diferentes tratamientos.

Table 6. Average mineralization index (IM) and CBM/COT ratio for the different treatments.

\begin{tabular}{c|c|c|c|c}
\hline \multirow{2}{*}{$\begin{array}{c}\text { Trata- } \\
\text { mientos }\end{array}$} & \multicolumn{2}{|c|}{ C-BM/COT } & \multicolumn{2}{c}{ IM } \\
\cline { 2 - 5 } & Inicio & Final & Inicio & Final \\
\hline $\mathrm{N}_{0}$ & 6,4 & 5,3 & 2,2 & 1,5 \\
$\mathrm{Fi}$ & 7,4 & 6,0 & 2,8 & 1,7 \\
$\mathrm{E}$ & 5,4 & 7,6 & 2,2 & 2,1 \\
\hline
\end{tabular}


fenológica (emergencia de la planta hasta la formación de las primeras hojas internas). En la segunda fase, desde la aparición de las primeras hojas internas hasta el final del ciclo, abarca los 30 días finales del cultivo y es donde se absorbe el $50 \%$ de los nutrientes totales y tiene lugar la mayor producción de materia seca (Maroto, 2002). En el ensayo, el agregado de la segunda dosis del fertilizante inorgánico correspondería a la fase de mayor consumo de nutrientes con la mayor disponibilidad de nitratos en los tratamientos Fi y E. Consecuentemente, en la tercera fecha de muestreo se produce un marcado descenso de nitratos en el tratamiento E (Cuadro 2). Según Frioni, (2006) el contenido de nitrógeno agregado es consumido en esas etapas fenológicas, parte se pierde por lavado o es inmovilizado por la biota del suelo.

\section{Producción del cultivo}

El aumento en la concentración de nitratos en la solución del suelo en las parcelas fertilizadas originó un incremento en la producción, siendo aún mayor en las parcelas con estiércol (Cuadro 3). Ello es de esperar, ya que la aplicación de estiércol es una fuente importante de agregado de otros nutrientes tales como fósforo, potasio, calcio y micronutrientes necesarios para el cultivo, como así también favorece las propiedades físicas del suelo (Sasal et al., 2000).

Se obtuvo un incremento en el contenido de nitrógeno en la biomasa foliar, aunque no se manifestó en los restantes macronutrientes ( $\mathrm{P}, \mathrm{Ca}$ y K). Estos nutrientes son poco móviles en el suelo y quedan disponibles para sucesivos cultivos a diferencia del $\mathrm{N}$ que se lixivia fácilmente con los riegos sin que sea aprovechado por la planta y al agregar fertilizantes es lógico esperar que se manifieste una mayor absorción.

\section{Eficiencia de uso del nitrógeno (EUN)}

La EUN se manifiesta a través de la cantidad de MS foliar producida. El incremento obtenido en el tratamiento E puede atribuirse a las características del material incorporado; un compuesto orgánico con relación de $\mathrm{C} / \mathrm{N}$ intermedia conduce a la inmovilización del $\mathrm{N}$, en especial aquellos compuestos carbonados de la viruta de madera utilizada en la cama de pollo que contiene una elevada proporción de lignina y celulosa. Los procesos de descomposición y mineralización del nitrógeno del estiércol son lentos (Smith y Peterson, 1982), esto permite que los nitratos sean mejor aprovechados en las distintas etapas fenológicas del cultivo.

El manejo del agua de riego y la distribución de fertilizantes influyen significativamente en la eficiencia del uso del nitrógeno (Thompson y Doerge, 1996). La baja eficiencia obtenida en este ensayo se atribuye en parte al lavado del nitrógeno con el riego. De acuerdo a las condiciones climáticas de la estación de crecimiento (primavera) durante el ciclo de cultivo, la lechuga necesita $280 \mathrm{~mm}$ (FAO, 1976) y la lámina de agua aplicada en el momento del transplante, en noviembre y cerca de cosecha fue aproximadamente de 180, 208 y $230 \mathrm{~mm}$ respectivamente. Esto demuestra la disminución brusca del contenido de nitratos en las parcelas fertilizadas y el manejo ineficiente del riego que realiza el productor. Roorda van Eysinga y van der Meijs (1985) señalan la dificultad de ajustar las cantidades de $\mathrm{N}$ a aportar en plantaciones comerciales, debido a la variabilidad en la mineralización del $\mathrm{N}$ procedente de la materia orgánica del suelo en relación al manejo del agua.

Los nitratos en el tratamiento E se distribuyeron significativamente hasta los $60 \mathrm{~cm}$ de profundidad (Cuadro 2). Es importante considerar que desde la incorporación del estiércol al transplante pasó suficiente tiempo (38 días) y los nitratos liberados superaron en ese lapso al consumo del cultivo en la primera fase de crecimiento, por lo cual parte se pierde por lavado y alcanzan los estratos subsuperficiales del suelo donde las raíces de la lechuga no aseguran su absorción. Se podría incrementar la eficiencia si el agregado del estiércol se realizara más próximo a la fecha de transplante.

El índice de eficiencia fisiológica (EF) representa la capacidad de las plantas en asimilar el $\mathrm{N}$ absorbido para producir materia seca total, es característico de cada planta y depende de factores externos (Hofman y van Cleemput, 2004). Cuando el contenido de $\mathrm{N}$ absorbido 
es mayor, aumenta el rendimiento, esto puede observarse en los tratamientos E y Fi (Cuadro 4).

\section{Variables biológicas}

La relación $\mathrm{C} / \mathrm{N}$ refleja el equilibrio dinámico de la actividad microbiana, los valores obtenidos fueron de 16,0 al inicio y 12,4 al final del ensayo, respectivamente. Dichas relaciones son consideradas bajas (Foth, 1997), produciéndose en consecuencia, una mineralización neta del nitrógeno. Los valores indican que existe una cantidad suficiente de nitrógeno en el sustrato para que los microorganismos conviertan el carbono en biomasa y el nitrógeno excedente sea utilizado por el cultivo (Jackson et al., 1989), Según Albanesi (2001) las prácticas convencionales tienden a homogeneizar la variabilidad del COT y por ende la relación $\mathrm{C} / \mathrm{N}$.

La respiración microbiana es la señal más evidente de la mineralización en el suelo (Frioni, 2006). En nuestro ensayo, el agregado de nitrógeno ya sea orgánico o mineral produjo un incremento en el C-BM y en la RE en los tratamientos Fi y E debido probablemente a la disponibilidad de $\mathrm{N}$ proveniente de materiales de fácil descomposición (Loveland y Weeb, 2003). Los índices de calidad de suelo que relacionan las variables químicas y biológicas, son representativos de la dinámica y el balance de los procesos biológicos del suelo (van Bruggen y Semenov, 2000; Filip, 2002). El índice (qCO2) de acuerdo a Anderson y Domsch, (1993) se relaciona con la hipótesis de optimización energética en ecosistemas en desarrollo y equivale a la respiración por unidad de biomasa. Debe ser elevado en ecosistemas jóvenes y bajo en los maduros. En los suelos degradados existe menor actividad microbiana y los valores de $\mathrm{qCO}_{2}$ son mayores, indicando ecosistemas inmaduros. En el suelo hortícola estudiado, los valores de $\mathrm{qCO}_{2}$ no variaron en los suelos fertilizados y sin fertilizar, indicando un ecosistema estable y maduro.

En cuanto al IM es recomendable hacer mediciones posteriores de este índice para determinar el tiempo requerido para lograr un adecuado equilibrio en el balance de mineralización y humificación de estos suelos, caso contrario afectaría la fertilidad y calidad del mismo, debido a un gasto de carbono orgánico.
De acuerdo a los resultados obtenidos en este ensayo los rendimientos y la eficiencia de utilización del nitrógeno fueron mayores en las parcelas fertilizadas con estiércol. Asimismo se manifestó un incremento en el C-BM. En cambio, en la combinación de las variables biológicas con las químicas no se obtuvo diferencia entre la fertilización mineral y la enmienda orgánica.

Se debería continuar con la utilización del enmendante orgánico, ajustando momento de aplicación y cantidad de agua a fin de lograr mayor utilización de nitrógeno y evaluar la evolución de las variables biológicas a través del tiempo.

\section{AGRADECIMIENTOS}

Este trabajo fue desarrollado en el marco del Proyecto de Investigación PI 04/A082 “Dinámica de los nitratos en sistemas productivos del Alto Valle de Río Negro" financiado por la Secretaría de la Universidad Nacional del Comahue y la cooperación de la Estación Experimental Agropecuaria Alto Valle de Río Negro (INTA) - Argentina.

\section{BIBLIOGRAFIA}

ABRIL, A. 2003. ¿Son los microorganismos edáficos buenos indicadores de impacto productivo en los ecosistemas?. Ecología Austral 13:195-204.

ABRIL, A.; BUCHER, E.H. 2001. Overgrazing and soil carbon dynamics in the Western Chaco of Argentina. Appl. Soil Ecol. 16: 243-249.

ALBANESI, A. 2001. Parámetros de calidad biológica del suelo para evaluar degradación en función del uso de la tierra en una zona del chaco semiárido, Argentina. Tesis Magíster UBA, Buenos Aires, Argentina. 168p.

ANDERSON, J.P.E.; DOMSCH, K.H. 1978. A physiological method for the quantitative measurement of microbial biomass in soils. Soil Biol. Biochem. 10: 215-221.

ANDERSON, J. P. E.; DOMSCH, K. H. 1993. The metabolic quotient for $\mathrm{CO}_{2}\left(\mathrm{qCO}_{2}\right)$ as a specific activity parameters to assess the effects of environmental conditions, such as $\mathrm{pH}$, on the microbial biomass of forest soils. Soil Biol. Biochem. 25:393-395. 
ARSHAD, M.; MARTIN, S. 2002. Identefying critical limits for soil quality indicators in agroecosystems. Agr. Ecosyst. Environ. 88:153-160.

ARUANI, M.C.; SANCHEZ, E.; REEB, P.; AUN, E. 2007. Variación de la concentración de nitratos en un suelo franco limoso del Alto Valle de Río Negro. Rev. FCA UN Cuyo, 39: 25-33.

BREMMER, A.E.; MULVANEY, C.S. 1982. Total nitrogen. In: Page A; Miller, R.H.; Keeney, D.R. (eds.). Methods of soil analysis. Part 2. ASA and SSSA, Madison, W. I. pp 595-624.

CANTLIFFE, D.J.; HOCHMUTH G.J.; KARCHI I.; SECKER, I.; BEN-YEHOSHUA, S. 1998. Nitrogen fertility requeriment for iceberg lettuce grow on sandland with plastic mulch and drip irrigation. Proc. Fla. State. Hort. Soc. 110: 306-309.

CARTER, M.R. 1991. The influence of the proportion of organic carbon and nitrogen in the microbial biomass of medium textured soils in a humid climate. Biol. Fertil. Soils 11:135-139.

COOPERBAND, L. 2000. Sustainable use of byproducts in land management. In: Barteks J M.; Dick, W.A. (eds.). Land Application e products of Agricultural, Industrial and Municipal By -Products. SSSA Book series $N^{\circ}$ 6, Madison, WI. USA. pp:215-235.

DRAGHI, L.M. 2005. Mecanización del huerto frutal: Tránsito controlado con vehículos de bajo peso y altas intensidades de tráfico. Reología del suelo agrícola de bajo tránsito. Modificaciones físicomecánicas del suelo vinculadas a la compactación debida al tráfico agrario. Universidad Nacional de la Plata. pp 85-106.

ECHEVERRÍA, H.E.;VIDELA C.C. 1998. Eficiencia fisiológica y de utilización de nitrógeno en trigo en la región pampeana Argentina. Ciencia del Suelo 16: 83-87.

ECHEVERRÍA, H.E.; SAINZ ROZAS, H.R. 2001. Eficiencia de recuperación del nitrógeno aplicado al estadio de seis hojas del maíz bajo riego en siembra directa y labranza convencional. Ciencia del Suelo 19: 57-66.

FAO. 1976. Riego y drenaje. Las necesidades de agua de los cultivos. Organización de las Naciones Unidas para la Agricultura y la Alimentación. Roma. 193 p.

FRIONI, L. 1999. Procesos microbianos. Editorial Fundación de la Universidad Nacional de Río Cuarto, Argentina, Tomo II. 286 p.

FRIONI, L. 2006. Microbiología: básica, ambiental y agrícola. Departamento de Publicaciones de la Facultad de Agronomía. Universidad de la
República. Montevideo, Uruguay. 407 p.

FILIP, Z.K. 2002. International approach to assessing soil quality by ecological parameters. Agr. Ecosyst. Environ. 88: 169-174.

FOTH, H.D. 1997. Fundamentos de la ciencia del suelo. México, Compañía Editorial Continental. $443 \mathrm{p}$.

GILI, P.; ARUANI, M.C. y AUN, E. 2007. Evaluación de algunos parámetros biológicos en suelos fertilizados con nitrógeno cultivados con manzano, en el Alto Valle de Río Negro. VI Reunión Nacional Científico Técnica de Biología del Suelo. VI Encuentro sobre fijación biológica de nitrógeno, Río Cuarto - Córdoba. Trabajo publicado en $\mathrm{CD}, 10 \mathrm{p}$.

HOFMAN, G.; VAN CLEEMPUT, O. 2004. Soil and Plant Nitrogen. International fertilizer industry association, París, Francia. pp 1-43.

IGLESIAS, N.; VAN KONIJNENBURG, A., RUIZ, C. 2002. Situación actual de la horticultura en la Norpatagonia. Fruticultura y Diversificación No 45 pp 31-37.

JACKSON, L.E.; SCHIMEL, J.P.; FIRESTONE, M.K. 1989. Short-term partitioning of ammonium and nitrate between plants and microorganisms in an annual grassland. Soil Biology and Biochemistry 21: 409-16.

JACKSON, L.E.; STIVERS, L.J.; WARDEN, B.T.; TANJI, K.K. 1993. Crop nitrogen utilization and soil nitrate loss in a lettuce field. Fertilizer Research 37: 93-105.

JOHNSON, J. L.; TEMPLE, K. L. 1964. Some variables affecting the measurement of "catalase activity” in soil. Soil Sci. Soc. Amer. Proc. 28: 207-209.

KARAM, F.; MOUNZER, O.; SARKIS, F.; LAHOUD, R. 2002. Yield and Nitrogen recovery of lettuce under different irrigations regimes. J. Appl. Hort. 4: 70:76.

LAL R. 1998. Land use and soil management effects on soil organic carbon dynamics on Alfisols in Western Nigeria, pp. 109-126. In: Lal, R.; Kimble, J.M.; Follet, R.F.; Stewart, B.A. (eds.). Soil processes and carbon cycle. Boca Raton, CRC Press. pp: 109-126.

LITTEL, R.C.; MILLIKEN, G.A.; STROUP, W.W.; WOLFINGER, R.D., SCHABENGERGER, O., 2006. SAS for Mixed Models. Second Edition. Cary, NC. SAS Institute, USA. 813 p.

LOVELAND, P.; WEBB J. 2003. Is there a critical level of organic matter in the agricultural soil of temperate regions? A review. Soil and Tillage Research 70: 1-18.

LUPWAYI N, Z.; RICE, W.A.; CLAYTON, G.W. 
1998. Soil microbial diversity and community structure under wheat as influenced by tillage and crop rotation. Soil Biol Biochem. 30: 1733-1741.

MACHET, J.M.; PIERRE, D.; RECOUS, S.; REMY, J.C. 1987. Signification du coefficient reel d'utilisation et consequences pour la fetilisation azotee des cultures. C.R. Acad. Agric. Fr. 73: 3955.

MAROTO, J.V. 2002. Horticultura herbácea especial. $5^{\circ}$ Edición. Madrid Barcelona, Ediciones Mundi Prensa, 702 p.

Mc PHARLIN, I.R.; AYLMORE, P.M.; JEFFERY, R.C. 1995. Nitrogen requirements of lettuce under sprinkler irrigation and trickle fertigation on spear wood sand. J. Plant Nutrition 18: 219-241.

NELSON, D.; SOMMERS, L. 1982. Methods of soil analysis. In: Page, A. (ed.). Agronomy 9, ASA, SSSA. pp 539-579.

NOVOA, R.; LOOMIS, R.S. 1981. Nitrogen and plant production. Plant and Soil 58: 177-204.

ÖHLINGER, R. 1996. Soil respiration by titration. In: Methods in Soil Biology. Schinner F., Öhlinger R., E. Kandeler and R. Margesin (eds.). Berlin, Springer-Verlag, pp. 95-98.

OLSEN, S.R.; COLE, C.V.; WATANABE, F.S.; DEAN L.A. 1.954. Estimation of available phosphorus in soil by extraction with sodium bicarbonate. USDA. Washington DC., USA. Circular 939. 19 p

PAUL, E.A.; CLARK, F.E. 1996. Soil Microbiology and Biochemestry, $2^{\text {th }}$. Ed. San Diego Academic Press. Academic Press, 340 p.

ROORDA van EYSINGA, J.P.; VAN der MEIJS, M.Q. 1985. Effect of nitrogen nutrition and global radiation on yield and nitrate content of lettuce grown under glass. Commun. Soil Sci. Plant. Anal. 16: 1293-1300.
SASAL, C.; ANDRIULO, A.; ULLE, J.; ABREGO, F.; BUENO, M. 2000. Efecto de diferentes enmiendas sobre algunas propiedades edáficas en sistemas de producción hortícola del centro norte de la región pampaeana húmeda. Ciencia del Suelo 18 : 95-104.

SMITH, J.H.; PETERSON, J.R. 1982. Recycling or nitrogen through land application of agricultural, food processing and municipal wastes. In: Stevenson, F.J. (ed.). Nitrogen in Agricultural Soils. Series Agronomy N N 22. ASA, CSSA, SSSA, Madison, WI, USA. pp 791-831.

SOIL SURVEY STAFF, 1998. Keys to Soil Taxonomy. $8^{\text {th }}$ Ed. USDA. Natural Resources Conservation Service. Washington, D C, USA 326 p.

SORENSEN, J.N.; JOHANSEN, A.S.; POULSEN, N. 1994. Influence of growth conditions on the value of crisphead lettuce: 1 . Marketable and nutritional quality as affected by nitrogen supply cultivar and plant age. Plant-Foods-for-Human-Nutrition 46: 1-11.

THOMPSON, T.L.; DOERGE, T.A., 1996. Nitrogen and water interactions in subsurface trickle-irrigated lettuce. Agronomic, economic and environmental outcomes. Soil Sci. Soc. Am. J. 60: 168-173.

VANBRUGGEN, A.H.C.; SEMENOV, A.M. 2000. En search of biological indicators for soil health and disease suppressión. Appl Soil Ecol. 15:13-24.

WALKLEY, A.; BLACK, I.A. 1934. An examination of the Degthareff method for determining soil organic matter and proposed modification of the chromic acid titration method. Soil Sci. 37:28-29.

WEAVER, R.; BOTTOMLEY, P. 1994. Methods of soil analysis. Part 2: Microbiological and biochemical properties. SSSA Book Series 5, Madison, USA. 1121p. 